\title{
Integrated miRNA profiling and bioinformatics analyses reveal upregulated miRNAs in gastric cancer
}

\author{
CHEN YUAN $^{1}$, YUE ZHANG ${ }^{2}$, WENWEN TU $^{3}$ and YUSHENG GUO ${ }^{1}$ \\ ${ }^{1}$ Department of Emergency, Huashan Hospital, Fudan University, Shanghai 200040; ${ }^{2}$ Department of Gerontology, \\ Shanghai Jiaotong University Affiliated to Sixth People's Hospital, Shanghai 201499; ${ }^{3}$ Department of Cardiology, \\ Jingan District Zhabei Central Hospital, Shanghai 200040, P.R. China
}

Received April 8, 2018; Accepted April 15, 2019

DOI: $10.3892 / \mathrm{ol} .2019 .10495$

\begin{abstract}
Gastric cancer is one of the most common malignancies in China and exhibits a poor prognosis. The most significant challenge for gastric cancer treatment is the absence of early diagnostic biomarkers. MicroRNAs (miRNAs) are small non-coding RNAs, which possess clinical value in a number of different types of cancer. The current study identified 13 miRNAs (hsa-miR-22, hsa-miR-545, hsa-let-7i, hsa-miR-15b, hsa-miR-221, hsa-miR-196a, hsa-miR-20a, hsa-miR-196b, hsa-miR-93, hsa-miR-19a, hsa-miR-503, hsa-miR-106b and hsa-miR-18a) that were significantly overexpressed in GC, by analyzing 1,000 GC samples included in four public datasets, including GSE23739, GSE78091, GSE30070 and The Cancer Genome Atlas. Furthermore, it was revealed that the expression levels of these 13 miRNAs were significantly higher in gastric cancer tissues of grades I, II and III compared with normal controls. Gene ontology analysis and Kyoto Encyclopedia of Genes and Genomes analysis demonstrated that the differentially expressed miRNAs were involved in regulating transcription, protein amino acid phosphorylation, signal transduction, protein binding, zinc ion binding, the mitogen-activated protein kinase signaling pathway and focal adhesion. In summary, the present study may provide potential new therapeutic and prognostic targets for gastric cancer.
\end{abstract}

\section{Introduction}

A number of studies have indicated that non-coding RNAs can serve as important regulators of biological processes (1). MicroRNAs (miRNAs) are small non-coding RNAs that are 18-25 nucleotides long, which are important post-transcriptional regulators of gene expression by inhibiting the stability

Correspondence to: Dr Chen Yuan, Department of Emergency, Huashan Hospital, Fudan University, 12 Wulumuqi Zhong Road, Shanghai 200040, P.R. China

E-mail: cyuan579@gmail.com

Key words: microRNA, gastric adenocarcinoma, bioinformatics analysis, expression profiling or translational efficiency of target genes (2). Recently studies have demonstrated that miRNAs are aberrantly expressed in different types of human cancer and serve important roles in regulating cancer initiation and progression (3). miRNAs can regulate numerous biological processes, including cancer cell proliferation, cycle arrest, apoptosis, migration and invasion (4).

Gastric cancer (GC) is one of the most common malignancies in China and exhibits a poor prognosis (5). A significant challenge for GC treatment is the absence of early diagnostic biomarkers (6). Yuan et al (7), reported that the 5-year survival rate of early GC is $\sim 30$-fold high compared with that of late-stage GC. A number of studies have identified aberrantly expressed miRNAs in GC, which serve regulatory roles in tumors (8). For example, miR-5590-3p has been revealed to inhibit GC growth by targeting the DDX5/AKT/mammalian target of rapamycin (mTOR) pathway (9). miRNA-223 promotes GC metastasis by targeting EPB41L3 (10). Additionally, miR-20a has been identified to be involved in the resistance of GC by targeting CYLD (11). However, these studies only investigated a small number of miRNAs and didn't perform a systems-level identification of differentially expressed miRNAs with a large sample size. Therefore, there is a requirement to understand the molecular mechanisms of miRNAs that regulate GC progression and identify miRNAs that may serve as biomarkers for the detection of GC.

The present study identified differentially expressed miRNAs in GC by analyzing four public datasets, including GSE23739, GSE26295, GSE30070 and data from The Cancer Genome Atlas (TCGA). In total, >800 GC samples were included in the present study. Additionally, to investigate the molecular mechanisms of aberrantly expressed miRNAs, gene ontology (GO) and Kyoto Encyclopedia of Genes and Genomes (KEGG) analysis were performed.

\section{Materials and methods}

Microarray data and data preprocessing. The current study screened GC-associated miRNA expression profiles in National Center for Biotechnology Information (NCBI) Gene Expression Omnibus (GEO) datasets (https://www. ncbi.nlm.nih.gov/gds/). Candidate datasets with $>50$ samples were selected for further analysis. Therefore, a total of four 
miRNA expression datasets of GC were downloaded from the NCBI GEO and TCGA (https://portal.gdc.cancer.gov/) databases including GSE23739, GSE30070, GSE26595 and data from TCGA. GSE23739 was submitted by Oh et al (12), and contained $40 \mathrm{GC}$ samples and 40 normal samples obtained from the National Cancer Centre and the Singhealth Tissue Repository (Singapore). GSE26595 was submitted by Lim et al (13), and contained 60 primary GC samples and 8 surrounding non-cancerous samples from patients who underwent a curative gastrostomy as a primary treatment method between 1999 and 2007 at Severance Hospital and Gangnam Severance Hospital, Yonsei University College of Medicine (Seoul, South Korea). GSE30070 was submitted by Kim et al (14), and contained 90 GC samples and 34 normal samples collected at the Hospital of Korean National Cancer Center by endoscopy between 2001 and 2006 following a protocol approved by the Institutional Review Board (IRB) of the National Cancer Center Hospital in Goyang, Korea. All patients and volunteers signed IRB-approved informed consent forms. A total of 34 healthy volunteers underwent gastroscopy for routine screening for GC and were confirmed to have normal gastric mucosa by histology. No gastritis was identified among the 34 healthy volunteers. TCGA data contained $389 \mathrm{GC}$ samples and 41 normal samples.

These datasets were based on different platforms. For example, GSE23739 was based on Agilent Human miRNA Microarrays and TCGA data were based on RNA-sequencing (RNA-seq). A previous study demonstrated that RNA-seq and microarray-based methods do not exhibit a high coincidence degree, although they are both good technologies for measuring gene expression level (15). The present study used TCGA data and three GEO datasets to identify common dysregulated miRNAs in GC. A similar analysis has also been reported by a number of other groups (16-19).

GO and KEGG pathway analysis. To predict the targets of the differentially expressed miRNAs, four different databases were used, including TargetScan (http://www. targetscan.org/), miRWALK (http://zmf.umm.uni-heidelberg. de/apps/zmf/mirwalk2/), miRDB (http://www.mirdb.org/) and starbase (http://starbase.sysu.edu.cn/). A total of 605 target mRNAs were obtained. Accordingly, the network between differentially expressed targets and miRNAs was constructed using Cytoscape v3.2.1 (http://www. cytoscape.org/) software. Molecular Annotation System 3.0 (http://bioinfo.capitalbio. $\mathrm{com} / \mathrm{mas} 3 /$ ) was used to determine the biological roles of the target mRNAs. Gene functions were classified into the following three subgroups: Biological process, molecular function and cellular component. The enriched GO terms were presented as enrichment scores. KEGG pathway analysis (https://www.genome.jp/kegg/pathway.html) was performed to determine the involvement of differentially expressed mRNAs in different biological pathways using DAVID system (https://david.ncifcrf.gov/tools.jsp). $\mathrm{P}<0.05$ was considered to indicate a statistically significant result.

Statistical analysis. Results were analyzed using SPSS software (version 15.0; SPSS, Inc.). Numerical data are presented as the mean \pm standard deviation. Statistical comparisons between two groups of normalized data were performed using
Table I. Analysis of four miRNA expression profiles, which include 579 gastric cancer samples and 123 normal samples.

\begin{tabular}{lccc}
\hline Dataset & Total cases & Normal cases & Tumor cases \\
\hline GSE23739 & 80 & 40 & 40 \\
GSE30070 & 124 & 34 & 90 \\
GSE26595 & 68 & 8 & 60 \\
TCGA & 430 & 41 & 389 \\
\hline
\end{tabular}

TCGA, The Cancer Genome Atlas.

a student's t-test or Mann-Whitney U-test according to the test condition. Statistical comparisons among multiple groups of normalized data were performed using one-way analysis of the variance followed by a Dunnett's post hoc test. $\mathrm{P}<0.05$ was considered to indicate a statistically significant difference.

\section{Results}

Identification of the significantly differentially expressed miRNAs in GC. To identify the significantly differentially expressed miRNAs in GC, the current study used four miRNA expression profiles including 579 GC samples and 123 normal samples, which were downloaded and analyzed from GEO and TCGA datasets, as presented in Table I and Fig. 1. A total of 10 miRNAs (hsa-miR-15b-5p, hsa-miR-21-5p, hsa-miR-93-5p, hsa-let-7i-5p, hsa-miR-25-3p, hsa-miR-185-5p, hsa-miR-181b-5p, hsa-miR-224-5p, hsa-miR-196b-5p and hsa-miR-135b-5p) were identified as upregulated and hsa-miR-204-5p was revealed as downregulated in GC in the four datasets. Clustering analysis was subsequently performed for all abnormally expressed miRNAs in GC (Fig. 1E and F; Table II).

Differentially expressed miRNAs are dysregulated in all stages of GC. To evaluate the possible prognostic value of the 11 abnormally expressed miRNAs, the present study analyzed RNA-seq data from TCGA, which included a cohort of 41 normal tissues, 7 grade I GC tissues, 140 grade II GC tissues and 232 grade III GC tissues (20). All patients were staged using the tumor-node-metastasis classification of the American Joint Committee on Cancer/International Union Against Cancer from 2009 (21). It was identified that the expression levels of hsa-miR-15b-5p, hsa-miR-21-5p, hsa-miR-93-5p, hsa-let-7i-5p, hsa-miR-25-3p, hsa-miR-185-5p, hsa-miR-181b-5p, hsamiR-224-5p, hsa-miR-196b-5p and hsa-miR-135b-5p were significantly upregulated, and hsa-miR-204-5p expression was significantly downregulated in grade I, II and III 3 GC tissues compared with the normal controls (Fig. 2A-K). However, differential expression levels of the miRNAs were not observed between the different GC stages. This analysis demonstrated that the abnormally expressed miRNAs may be associated with GC progression and may serve as early diagnostic biomarkers. However, the dysregulation of these miRNAs could not predict the stage of GC.

Construction of a differentially expressed miRNAs-mRNAs network for GC. Four different databases were used, including 

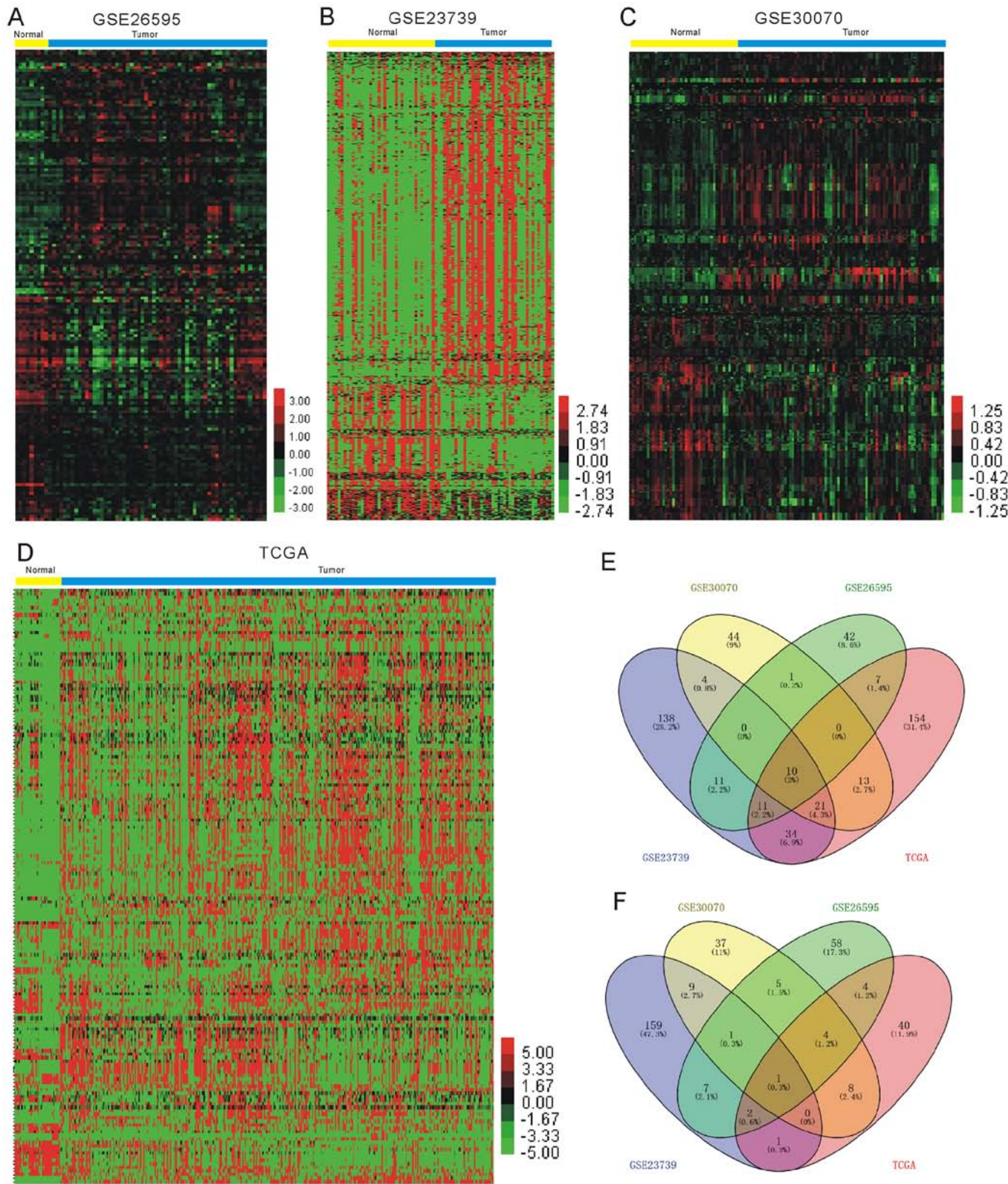

E
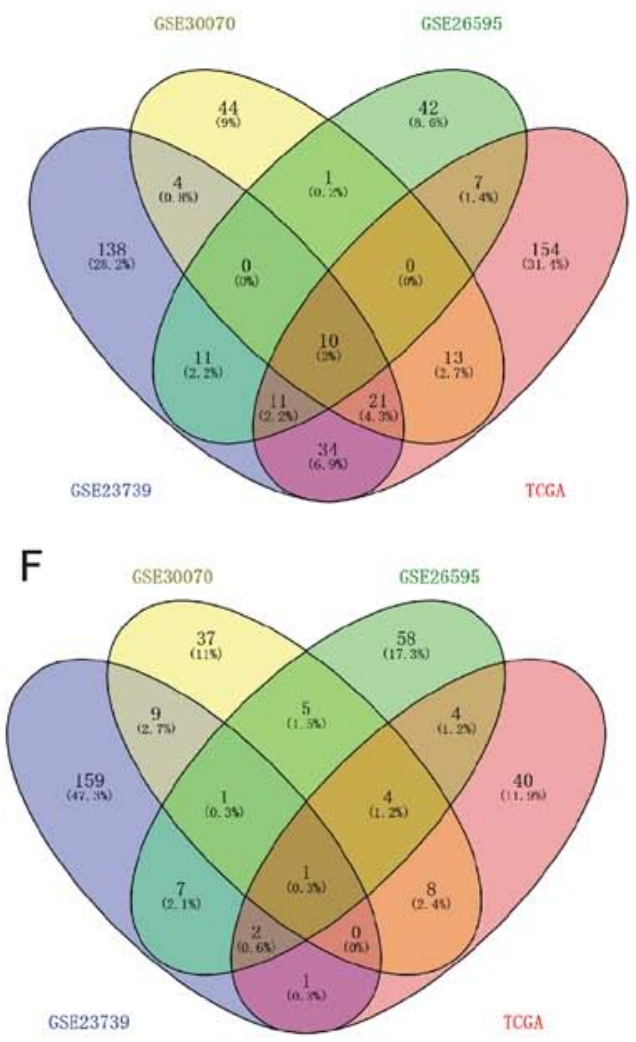

Figure 1. Identification of the significantly differentially expressed miRNAs in gastric cancer. Heatmaps of the differentially expressed miRNAs in (A) GSE26595, (B) GSE23739, (C) GSE30070 and (D) TCGA data. Clustering analysis of the (E) upregulated miRNAs and (F) downregulated miRNAs in the four datasets. miRNA, microRNA; TCGA, The Cancer Genome Atlas.

TargetScan, miRWALK, miRDB and starbase. A total of 605 target mRNAs were obtained by combining the analyses of these databases (Fig. 3A). Furthermore, the TCGA dataset was analyzed to investigate the expression pattern of the 605 target mRNAs in GC (Fig. 3B). In the present study, the downregulated genes were selected as potential targets of the upregulated miRNAs and the upregulated genes were selected as potential targets of the downregulated miRNAs. As presented in Fig. 3C, a total of 11 miRNAs and 267 mRNAs were included in this network. Four miRNAs, 
including hsa-miR-93-5p, hsa-miR-15b-5p, hsa-let-7i-5p and hsa-miR-204-5p were identified as key regulators in GC as they were connected to $>40$ mRNAs in the network.

GO analysis of differentially expressed miRNAs. GO analysis was performed for the differentially expressed miRNAs using the target mRNAs. We hypothesized that the potential functions of target mRNAs could reflect the possible roles of differentially expressed miRNAs. The present study only presented the GO analysis results for the biological processes and molecular functions (Fig. 4). According to the GO analysis, differentially expressed miRNAs were enriched in 'positive regulation of transcription', 'anterior/posterior pattern specification', 'proteasome-mediated ubiquitin-dependent protein catabolic process', 'embryonic hemopoiesis', 'positive regulation of G1/S transition of mitotic cell cycle', 'canonical Wnt signaling pathway', 'regulation of cyclin-dependent protein serine/threonine kinase activity', 'regulation of apoptotic process' and 'positive regulation of small GTPase mediated signal transduction' (Fig. 4A).

In addition, the differentially expressed miRNAs were involved in regulating several molecular functions, including 'receptor signaling protein serine/threonine kinase activity', 'ATP binding', 'transcription factor activity', 'sequence-specific DNA binding', 'sequence-specific DNA binding' and 'coreceptor activity' (Fig. 4B).

KEGG analysis of differentially expressed miRNAs. KEGG pathway analysis revealed that the differentially expressed miRNAs predominantly participate in the regulation of 'Insulin resistance', 'Transcriptional misregulation in cancer', 'FoxO signaling pathway', 'mTOR signaling pathway', 'Glucagon signaling pathway', 'Neurotrophin signaling pathway' and 'cGMP-PKG signaling pathway' (Fig. 4C).

Notably, it was identified that the differentially expressed miRNAs serve crucial roles in regulating the forkhead box protein $\mathrm{O}$ (FoxO) signaling pathway and the mTOR signaling pathway. Nine genes in the FoxO signaling pathway, including AKT3, FBXO32, CCND2, CCNG2, PTEN, PRKAB2, SGK1, STAT3 and TGFBR2, and six genes in the mTOR signaling pathway, including AKT3, RICTOR, EIF4B, PTEN, RPS6KA3 and RPS6KA6, were identified to be targets of the differentially expressed miRNAs (Fig. 5).

\section{Discussion}

The most significant challenge for the treatment of GC is the absence of early diagnostic biomarkers (1). miRNAs are small non-coding RNAs that demonstrate clinical value in a number of types of cancer (2). Numerous studies have identified certain differentially expressed miRNAs in GC (22-24); however, these studies only investigated a small number of miRNAs and lacked large sample sizes to systemically identify numerous differentially expressed miRNAs.

Previous studies regarding GC have indicated that hsa-miR-93 (25), hsa-miR-19a (26), hsa-miR-20a (27) and hsa-miR-221 (28) might act as oncogenic miRNAs. To identify differentially expressed miRNAs in GC, the present study analyzed four public datasets, including GSE23739, GSE26595, GSE30070 and data from TCGA. 

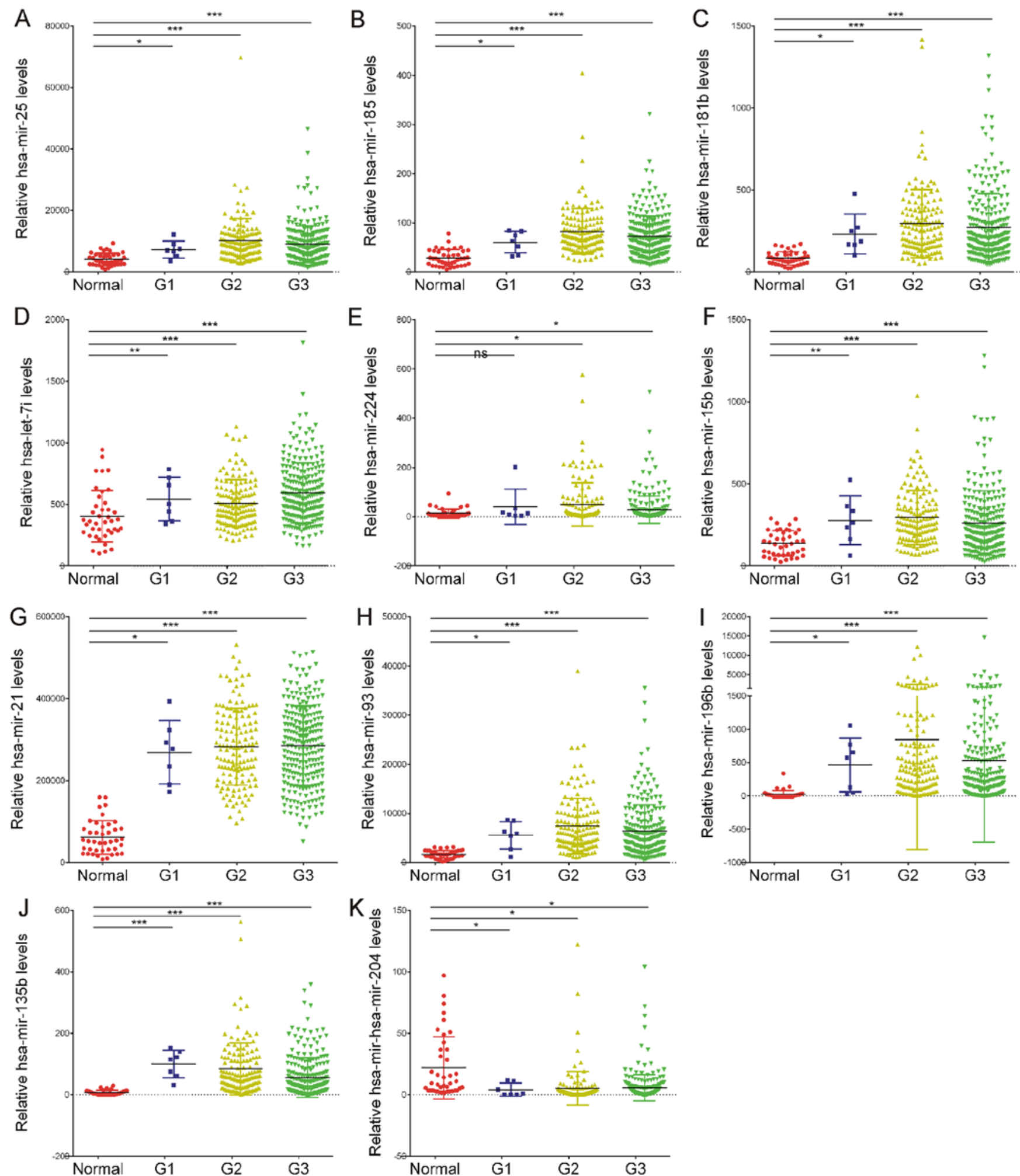

Figure 2. Dot-plot assay for the expression levels of the 11 abnormally expressed miRNAs in G1, G2 and G3 gastric cancer tissues compared with the normal controls. (A) hsa-miR-25-3p, (B) hsa-miR-185-5p, (C) hsa-miR-181b-5p, (D) hsa-let-7i-5p, (E) hsa-miR-224-5p, (F) hsa-miR-15b-5p, (G) hsa-miR-21-5p, (H) hsa-miR-93-5p, (I) hsa-miR-196b-5p and (J) hsa-miR-135b-5p were identified to be significantly upregulated in G1, G2 and G3 gastric cancer tissues compared with the normal samples. (K) hsa-miR-204-5p was revealed to be downregulated in G1, G2 and G3 gastric cancer tissues compared with the normal samples. ${ }^{*} \mathrm{P}<0.05,{ }^{* *} \mathrm{P}<0.01,{ }^{* * *} \mathrm{P}<0.001$. miRNA or miR, microRNA; G1, grade I; G2, grade II; G3, grade III.

A total of 10 miRNAs (hsa-miR-15b-5p, hsa-miR-21-5p, hsamiR-93-5p, hsa-let-7i-5p, hsa-miR-25-3p, hsa-miR-185-5p, hsa-miR-181b-5p, hsa-miR-224-5p, hsa-miR-196b-5p and hsa-miR-135b-5p) were revealed to be upregulated in GC and hsa-miR-204-5p was identified to be downregulated in GC, which suggests these miRNAs serve key roles in GC. To evaluate the possible prognostic value of these abnormally expressed miRNAs, the current study analyzed RNA-seq 

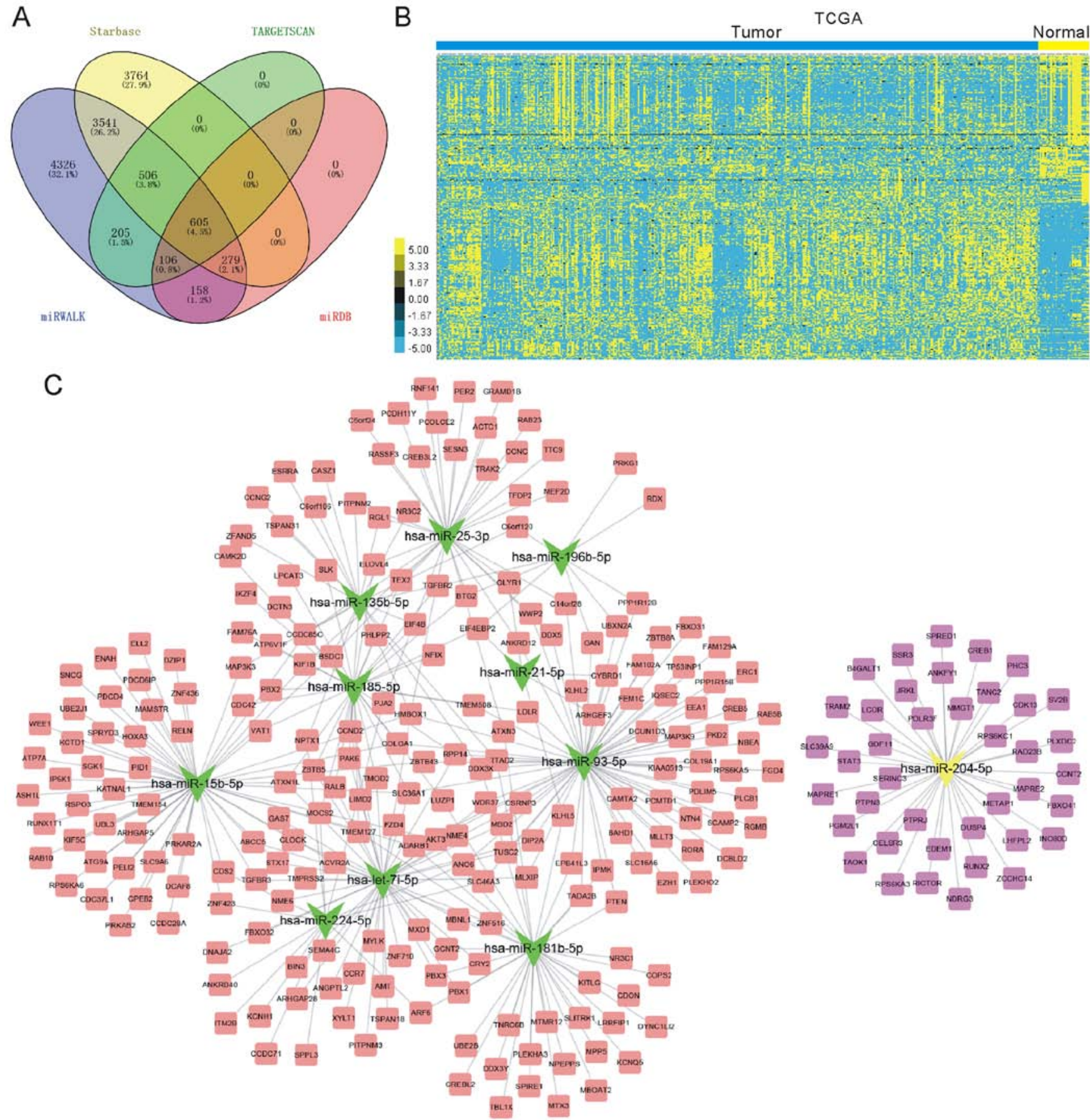

Figure 3. Prediction of targets of the upregulated miRNAs. (A) Clustering analysis of the predicted targets of 11 abnormally expressed miRNAs by four databases including TargetScan, miRWALK, miRDB and starbase. (B) Analysis of the expression patterns of the 605 target mRNAs in GC using the TCGA database. (C) A network between the targets and differentially expressed miRNAs was constructed using Cytoscape. Green nodes, upregulated miRNAs; red nodes, target mRNAs of upregulated miRNAs; yellow nodes, downregulated miRNAs; purple nodes, target mRNAs of downregulated miRNAs. TCGA, The Cancer Genome Atlas; miRNA or miR, microRNA.

data from TCGA and identified that the expression levels of the miRNAs were significantly upregulated or downregulated in grade I, II and III GC tissues compared with the normal samples. However, differential expression levels of the miRNAs were not observed between the different GC stages. This analysis demonstrated that these abnormally expressed miRNAs may be associated with GC progression and may serve as diagnostic biomarkers. Furthermore, previous studies have indicated that hsa- hsa-miR-93 (25), hsa-miR-19a (26), hsa-miR-20a (27) and hsa-miR-221 (28) may act as oncogenic
miRNAs in GC, which is consistent with the current analysis. The current study performed a comprehensive analysis to identify key miRNAs in GC progression by using a series of public datasets. The results may assist with the identification of biomarkers for the prognosis of GC.

Considering the important roles of miRNAs in human disease, a number of studies have investigated the functions and molecular mechanisms of miRNAs by performing loss or gain of function assays (29-32). However, these experimental validations are expensive and time-consuming. Therefore, 


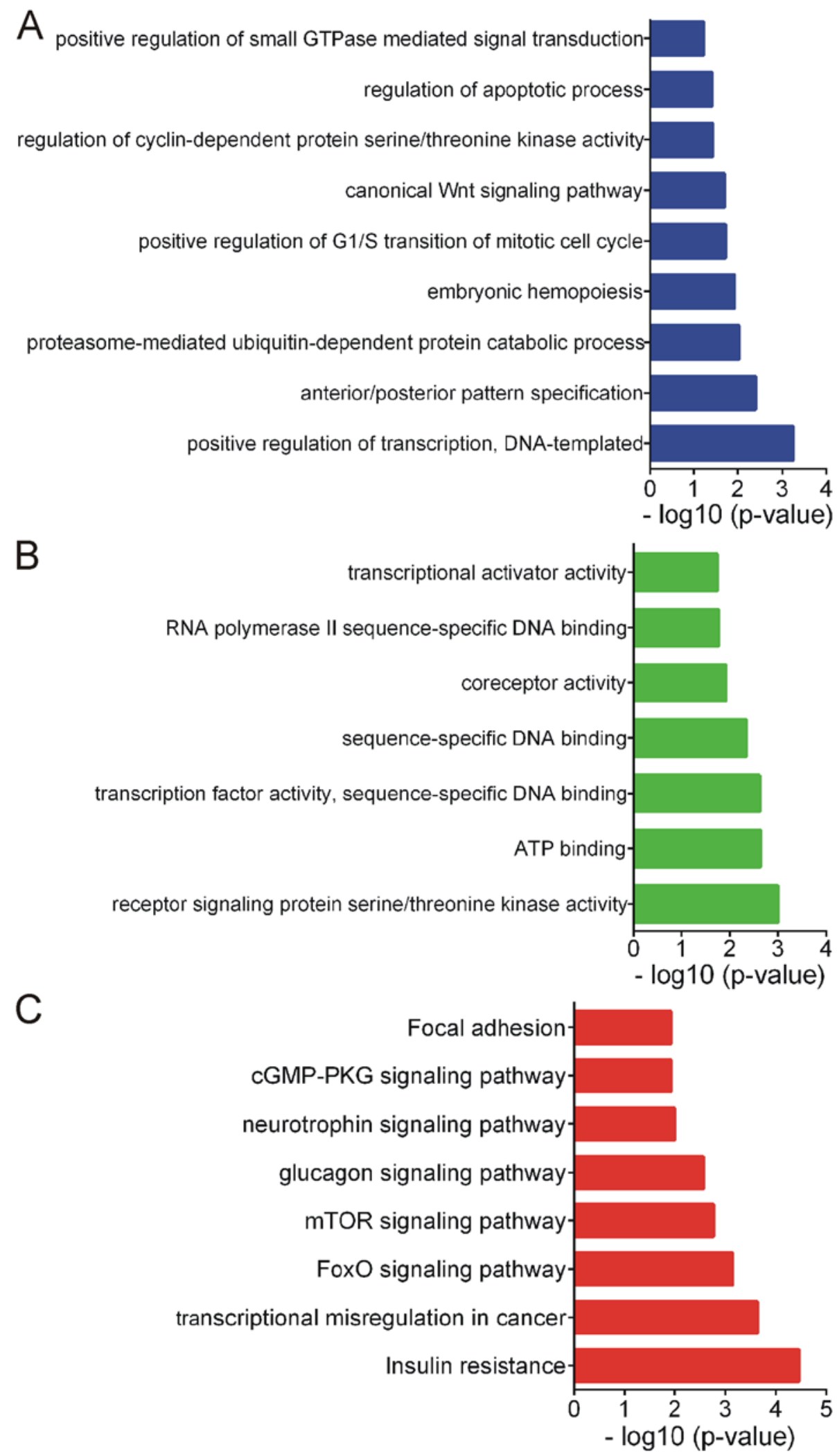

Figure 4. GO and KEGG analysis of the target mRNAs of differentially expressed miRNAs. (A) Biological processes analysis of the differentially expressed miRNAs using the target mRNAs. (B) Molecular function analysis of the differentially expressed miRNAs by using the target mRNAs. (C) KEGG pathway analysis of the differentially expressed miRNAs. The bioinformatics analyses were performed using Molecule Annotation System. GO, gene ontology; KEGG, Kyoto Encyclopedia of Genes and Genomes; miRNA, microRNA.

a number of effective and feasible computational methods have been developed to assist with the prediction of potential associations between disease and miRNAs. For example,
Chen et al (33) developed the Path-Based miRNA-Disease Association model for miRNA-disease association prediction. In addition, Chen et al $(33,34)$ developed the Bipartite 
A

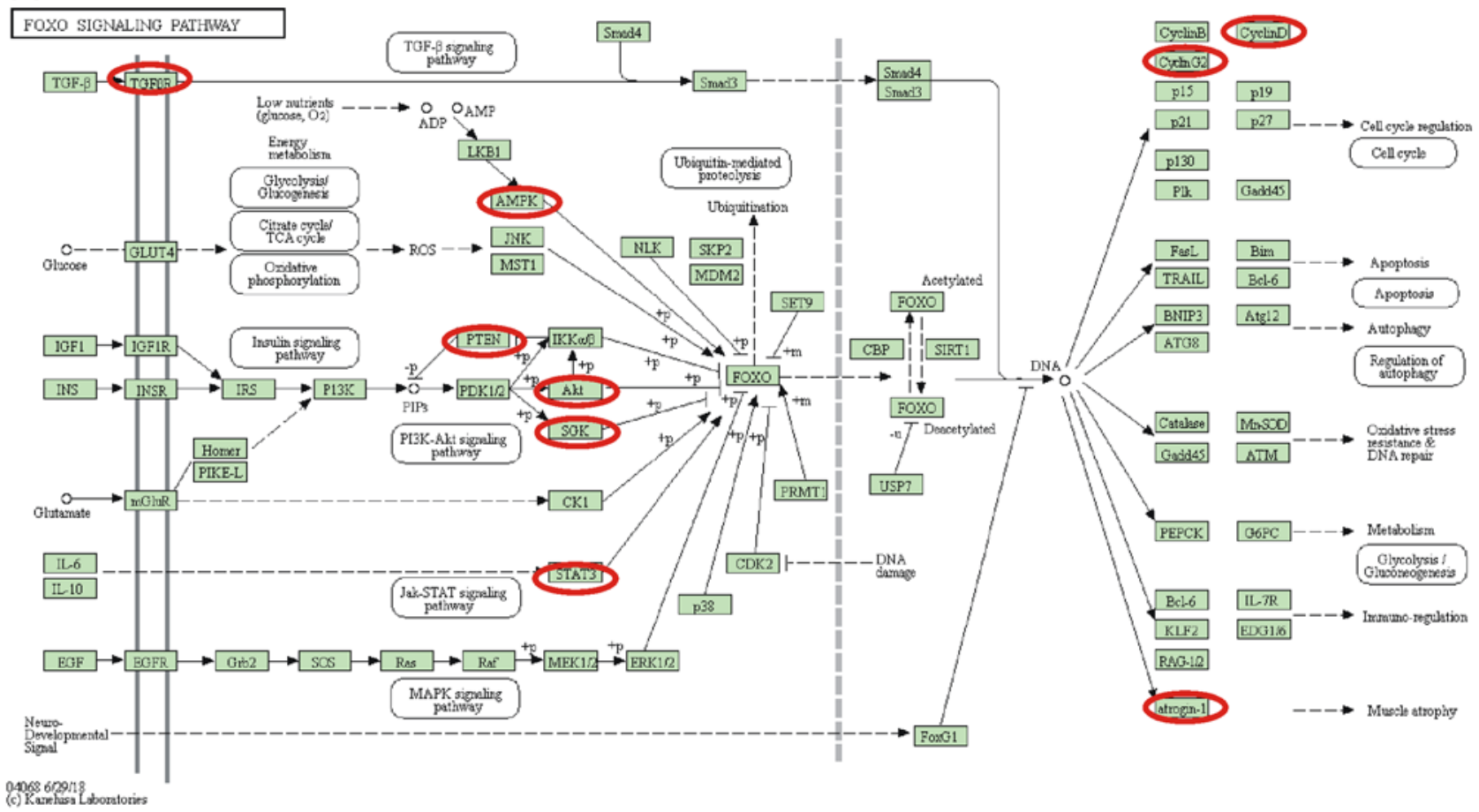

B

mTOR SIGNALING PATHWAY

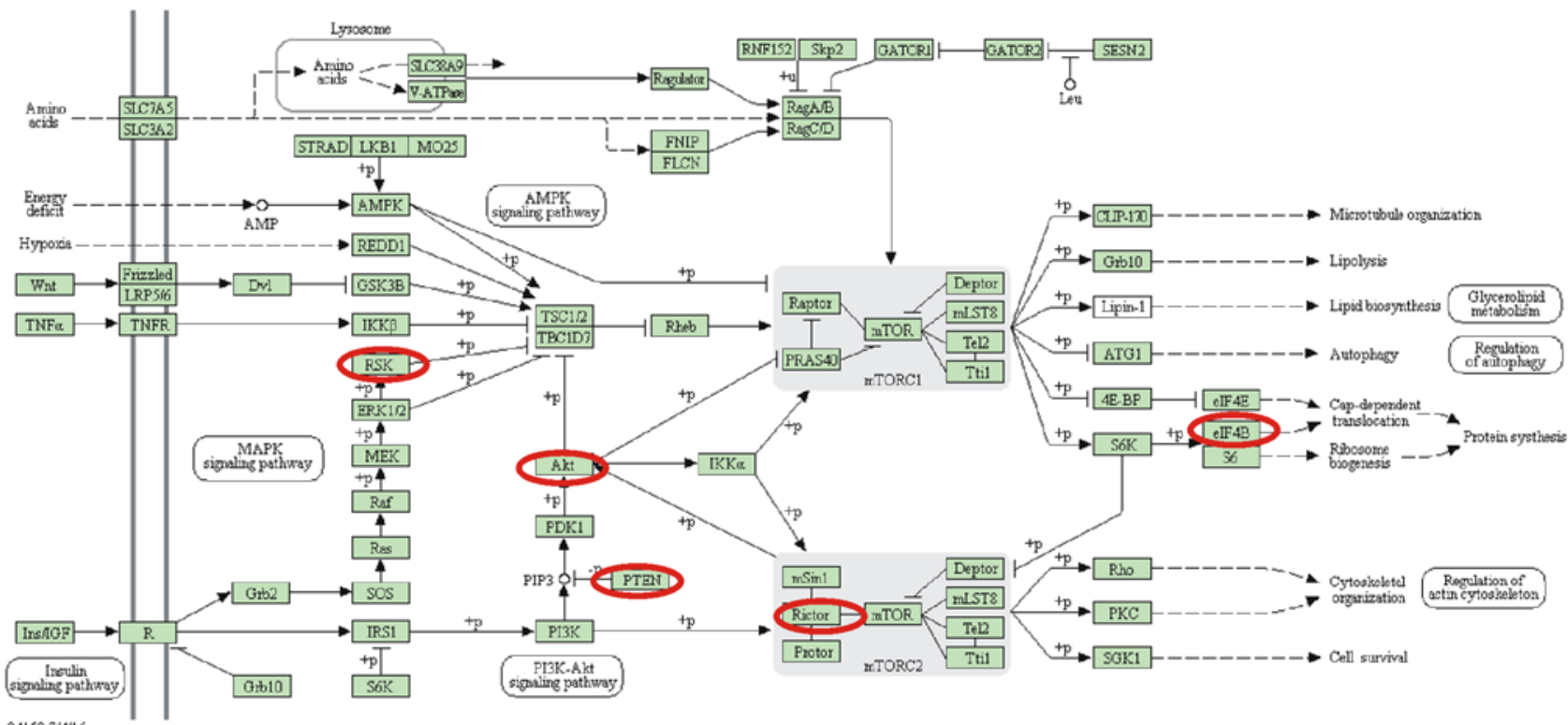

0415074416
(c) Kantilia Laboratones

Figure 5. Key Kyoto Encyclopedia of Genes and Genomes pathways associated with the differentially expressed miRNAs. Gene targets of the differentially expressed miRNAs that are associated with the (A) FoxO and (B) mTOR signaling pathways are indicated by a red circle. miRNA, microRNA; FoxO, forkhead box O; mTOR, mammalian target of rapamycin.

Network Projection for miRNA-Disease Association and Hybrid Approach for miRNA-Disease Association prediction models for miRNA-disease prediction. It can be suggested that a combination of experimental validations, and effective and feasible computational prediction methods is a useful strategy to investigate the potential roles of miRNAs in human disease. The present study constructed a differently expressed miRNAs-mRNAs network, and performed GO and KEGG analysis with the target mRNAs. A total of 11 miRNAs and
267 mRNAs were included in this network. Four miRNAs, including hsa-miR-93-5p, hsa-miR-15b-5p, hsa-let-7i-5p and hsa-miR-204-5p were identified as key regulators in GC as there were connected with $>40$ mRNAs in the network.

Bioinformatics analysis revealed that the differentially expressed miRNAs are associated with 'positive regulation of transcription', 'positive regulation of G1/S transition of mitotic cell cycle', 'canonical Wnt signaling pathway' and 'regulation of apoptotic process'. KEGG pathway 
analysis identified that the differentially expressed miRNAs predominantly participate in regulation of 'Insulin resistance', 'Transcriptional misregulation in cancer', 'FoxO signaling pathway', 'mTOR signaling pathway', 'Glucagon signaling pathway', 'Neurotrophin signaling pathway' and 'cGMP-PKG signaling pathway'. Notably, nine genes associated with the FoxO signaling pathway, including AKT3, FBXO32, CCND2, CCNG2, PTEN, PRKAB2, SGK1, STAT3 and TGFBR2, and six genes associated with the mTOR signaling pathway, including AKT3, RICTOR, EIF4B, PTEN, RPS6KA3 and RPS6KA6, were identified as targets of the differentially expressed miRNAs. Previous studies have demonstrated the important roles of FoxO and mTOR signaling in GC progression. For example, downregulation of FOXO4 was revealed to inhibit tumor proliferation and metastasis in GC (35). In addition, mTOR signaling serves crucial roles in regulating GC proliferation, apoptosis and metastasis $(36,37)$. Nadauld et al (38) identified that TGFBR2 acts an oncogene in diffuse GC. Furthermore, PTEN has been demonstrated to be involved in regulating apoptotic cell death, metastasis, angiogenesis and chemoresistance in GC (39). These results suggest that dysregulation of these miRNAs have important roles in GC progression.

In conclusion, the present study identified 11 miRNAs that are significantly differently expressed in GC by analyzing 1,000 GC samples from four public datasets, including GSE23739, GSE26595, GSE30070 and data from TCGA. hsa-miR-93-5p, hsa-miR-15b-5p, hsa-let-7i-5p and hsa-miR-204-5p were revealed as key regulators in GC. GO analysis and KEGG analysis demonstrated that the differentially expressed miRNAs are involved in the positive regulation of transcription, positive regulation of the G1/S transition in the mitotic cell cycle, the canonical Wnt signaling pathway, the FoxO signaling pathway and the mTOR signaling pathway. In summary, the current study may provide potential new therapeutic and prognostic targets for GC.

\section{Acknowledgements}

Not applicable.

\section{Funding}

No funding was received.

\section{Availability of data and materials}

All data generated or analyzed during the present study are included in this published article.

\section{Authors' contributions}

CY and YG conceived and designed the study. CY, YZ and WT developed the methodology. CY and YZ analyzed and interpreted the data. CY, YZ, WT and YG drafted, reviewed and revised the manuscript.

\section{Ethics approval and consent to participate}

Not applicable.

\section{Patient consent for publication}

Not applicable.

\section{Competing interests}

The authors declare that they have no competing interests.

\section{References}

1. Hudler P: Outlook on epigenetic therapeutic approaches for treatment of gastric cancer. Current Cancer Drug Targets 18: 65-88, 2018.

2. Ondracek J,Fadrus P, Sana J, Besse A, Loja T, Vecera M, Radova L, Smrcka M, Slampa P and Slaby O: Global MicroRNA expression profiling identifies unique MicroRNA pattern of radioresistant glioblastoma cells. Anticancer Res 37: 1099-1104, 2017.

3. Wan X, Pu H, Huang W, Yang S, Zhang Y, Kong Z, Yang Z, Zhao P, Li A, Li T and Li Y: Androgen-induced miR-135a acts as a tumor suppressor through downregulating RBAK and MMP11, and mediates resistance to androgen deprivation therapy. Oncotarget 7: 51284-51300, 2016.

4. Wu S, Gu Y, Huang Y, Wong TC, Ding H, Liu T, Zhang Y and Zhang $X$ : Novel biomarkers for non-functioning invasive pituitary adenomas were identified by using analysis of microRNAs expression profile. Biochem Genet 55: 253-267, 2017.

5. Chen W, Zheng R, Baade PD, Zhang S, Zeng H, Bray F, Jemal A, $\mathrm{Yu}$ XQ and He J: Cancer statistics in China, 2015. CA Cancer J Clin 66: 115-132, 2016.

6. Oze I, Shimada S, Nagasaki H, Akiyama Y, Watanabe M, Yatabe Y, Matsuo K and Yuasa Y: Plasma microRNA-103, microRNA-107, and microRNA-194 levels are not biomarkers for human diffuse gastric cancer. J Cancer Res Clin Oncol 143: 551-554, 2017.

7. Yuan M, Wang Z, Hu G, Yang Y, Lv W, Lu F and Zhong H: A retrospective analysis of hyperthermic intraperitoneal chemotherapy for gastric cancer with peritoneal metastasis. Mol Clin Oncol 5: 395-399, 2016.

8. Yuan X, Wang S, Liu M, Lu Z, Zhan Y, Wang W and Xu AM: Histological and pathological assessment of miR-204 and SOX4 levels in gastric cancer patients. Biomed Res Int 2017: 6894675, 2017.

9. Wu N, Han Y, Liu H, Jiang M, Chu Y, Cao J, Lin J, Liu Y, Xu B and $\mathrm{Xie} X$ : miR-5590-3p inhibited tumor growth in gastric cancer by targeting DDX5/AKT/m-TOR pathway. Biochem Biophys Res Commun 503: 1491-1497, 2018.

10. Li X, Zhang Y, Zhang H, Liu X, Gong T, Li M, Sun L, Ji G, Shi Y, Han Z, et al: miRNA-223 promotes gastric cancer invasion and metastasis by targeting tumor suppressor EPB41L3. Mol Cancer Res 9: 824-833, 2011.

11. Zhu M, Zhou X, Du Y, Huang Z, Zhu J, Xu J, Cheng G, Shu Y, Liu P, Zhu W and Wang T: miR-20a induces cisplatin resistance of a human gastric cancer cell line via targeting CYLD. Mol Med Rep 14: 1742-1750, 2016.

12. Oh HK, Tan ALK, Das K, Ooi CH, Deng NT, Tan IB, Beillard E, Lee J, Ramnarayanan K, Rha SY, et al: Genomic loss of miR-486 regulates tumor progression and the OLFM4 antiapoptotic factor in gastric cancer. Clin Cancer Res 17: 2657-2667, 2011.

13. Lim JY, Yoon SO, Seol SY, Hong SW, Kim JW, Choi SH, Lee JS and Cho JY: Overexpression of miR-196b and HOXA10 characterize a poor-prognosis gastric cancer subtype. World J Gastroenterol 19: 7078-7088, 2013.

14. Kim CH, Kim HK, Rettig RL, Kim J, Lee ET, Aprelikova O, Choi IJ, Munroe DJ and Green JE: miRNA signature associated with outcome of gastric cancer patients following chemotherapy. BMC Med Genomics 4: 79, 2011.

15. Li J, Hou R, Niu X, Liu R, Wang Q, Wang C, Li X, Hao Z, Yin $\mathrm{G}$ and Zhang K: Comparison of microarray and RNA-Seq analysis of mRNA expression in dermal mesenchymal stem cells. Biotechnol Lett 38: 33-41, 2016.

16. Wang J, Meng F, Dai E, Yang F, Wang S, Chen X, Yang L, Wang $\mathrm{Y}$ and Jiang W: Identification of associations between small molecule drugs and miRNAs based on functional similarity. Oncotarget 7: 38658-38669, 2016.

17. Liu H, Zhang Q, Lou Q, Zhang X, Cui Y, Wang P, Yang F, Wu F, Wang J, Fan T3 and Li S: Differential analysis of lncRNA, miRNA and mRNA expression profiles and the prognostic value of lncRNA in esophageal cancer. Pathol Oncol Res: Apr 10, 2019 (Epub ahead of print). 
18. He S, Shi J, Mao J, Luo X, Liu W, Liu R and Yang F: The expression of miR-375 in prostate cancer: A study based on GEO, TCGA data and bioinformatics analysis. Pathol Res Pract: 152375, 2019 (Epub ahead of print).

19. He K, Li W, Guan D, Gong M, Ye S, Fang Z, Huang JF and Lu A: Regulatory network reconstruction of five essential microRNAs for survival analysis in breast cancer by integrating miRNA and mRNA expression datasets. Funct Integr Genomics: Mar 12, 2019 (Epub ahead of print)

20. Yu H, Ye L, Mansel RE, Zhang Y and Jiang WG: Clinical implications of the influence of Ehm2 on the aggressiveness of breast cancer cells through regulation of matrix metalloproteinase-9 expressio. Mol Cancer Res 8: 1501-1512, 2010.

21. Edge SB and Compton CC: The American joint committee on cancer: The 7th edition of the AJCC cancer staging manual and the future of TNM. Ann Surg Oncol 17: 1471-1474, 2010.

22. Lu R, Zhao G, Yang Y, Jiang Z, Cai J, Zhang Z and Hu H: Long noncoding RNA HOTAIRM1 inhibits cell progression by regulating miR-17-5p/PTEN axis in gastric cancer. J Cell Biochem 120: 4952-4965, 2019.

23. Magalhães L, Quintana L, Lopes DCF, Vidal AF, Pereira AL, D'Araujo Pinto LC, de Jesus Viana Pinheiro J, Khayat AS, Goulart LR, Burbano R, et al: APC gene is modulated by hsa-miR-135b-5p in both diffuse and intestinal gastric cancer subtypes. BMC Cancer 18: 1055, 2018.

24. Liu R, Zhang C, Hu Z, Li G, Wang C, Yang C, Huang D, Chen X, Zhang H, Zhuang R, et al: A five-microRNA signature identified from genome-wide serum microRNA expression profiling serves as a fingerprint for gastric cancer diagnosis. Eur J Cancer 47: 784-791, 2011.

25. Liang H, Wang F, Chu D, Zhang W, Liao Z, Fu Z, Yan X, Zhu H, Guo W, Zhang Y, et al: miR-93 functions as an oncomiR for the downregulation of PDCD4 in gastric carcinoma. Sci Rep 6: 23772, 2016

26. Wu Q, Yang Z, An Y, Hu H, Yin J, Zhang P, Nie Y, Wu K, Shi Y and Fan D: MiR-19a/b modulate the metastasis of gastric cancer cells by targeting the tumour suppressor MXD1. Cell Death Dis 5: e1144, 2014

27. Zhang $Y$, Han $T$, Wei $G$ and Wang Y: Inhibition of microRNA-17/20a suppresses cell proliferation in gastric cancer by modulating UBE2C expression. Oncol Rep 33: 2529-2536, 2015.
28. Shi J, Zhang Y, Jin N, Li Y, Wu S and Xu L: MicroRNA-221-3p plays an oncogenic role in gastric carcinoma by inhibiting PTEN expression. Oncol Res 25: 523-536, 2017.

29. Zhang Z, Li Z, Gao C, Chen P, Chen J, Liu W, Xiao S and Lu H: miR-21 plays a pivotal role in gastric cancer pathogenesis and progression. Lab Invest 88: 1358-1366, 2008.

30. Wu H, Lin W and Tsai K: Advances in molecular biomarkers for gastric cancer: miRNAs as emerging novel cancer markers. Expert Rev Mol Med 16: e1, 2014.

31. Shin VY and Chu K: MiRNA as potential biomarkers and therapeutic targets for gastric cancer. World J Gastroenterol 20: 10432-10439, 2014.

32. Tchernitsa OI, Kasajima A, Schäfer R, Kuban RJ, Ungethüm U, Györffy B, Neumann U, Simon E, Weichert W, Ebert MP and Röcken C: Systematic evaluation of the miRNA-ome and its downstream effects on mRNA expression identifies gastric cancer progression. J Pathol 222: 310-319, 2010.

33. Chen X, Xie D, Wang L, Zhao Q, You Z and Liu H: BNPMDA: Bipartite network projection for MiRNA-disease association prediction. Bioinformatics 34: 3178-3186, 2018.

34. Chen X, Niu Y, Wang G and Yan G: HAMDA: Hybrid approach for MiRNA-disease association prediction. J Biomed Inform 76: 50-58, 2017.

35. Su L, Liu X, Chai N, Lv L, Wang R, Li X, Nie Y, Shi Y and Fan D: The transcription factor FOXO4 is down-regulated and inhibits tumor proliferation and metastasis in gastric cancer. BMC Cancer 14: 378, 2014.

36. Matsuoka T and Yashiro M: The role of PI3K/Akt/mTOR signaling in gastric carcinoma. Cancers (Basel) 6: 1441-1463, 2014.

37. Wu YJ, Wong BS, Yea SH, Lu CI and Weng SH: Sinularin induces apoptosis through mitochondria dysfunction and inactivation of the $\mathrm{pI} 3 \mathrm{~K} / \mathrm{Akt} / \mathrm{mTOR}$ pathway in gastric carcinoma cells. Mar Drugs 14: pii: E142, 2016

38. Nadauld LD, Garcia S, Natsoulis G, Bell JM, Miotke L, Hopmans ES, Xu H, Pai RK, Palm C, Regan JF, et al: Metastatic tumor evolution and organoid modeling implicate TGFBR2 as a cancer driver in diffuse gastric cancer. Genome Biol 15: 428, 2014.

39. Xu W, Yang Z and Lu N: Roles of PTEN (Phosphatase and Tensin Homolog) in gastric cancer development and progression. Asian Pac J Cancer Prev 15: 17-24, 2014. 